\title{
Hemoglobin S Polymerization Effect on Water Self-Diffusion Coefficient
}

\author{
Manuel A. Lores Guevara, Juan C. García-Naranjo, Yulianela Mengana, Jorge Pereira \\ Magnetic Resonance Lab, Biophysics and Medical Physics Centre, Santiago de Cuba, Cuba \\ Email: manuel.lores@cbiomed.cu
}

Received 26 July 2014; revised 13 September 2014; accepted 28 September 2014

Copyright @ 2014 by authors and Scientific Research Publishing Inc.

This work is licensed under the Creative Commons Attribution International License (CC BY). http://creativecommons.org/licenses/by/4.0/

c) (i) Open Access

\begin{abstract}
The transversal relaxation time, the effective transversal relaxation time and the water self-diffusion coefficient are evaluated during hemoglobin $S$ polymerization. One homogeneous permanent magnet and one inhomogeneous and portable unilateral magnet with a very strong and constant static magnetic field gradient were utilized. The Carr-Purcell-Meiboom-Gill method was used before and after placing the studied samples 24 hours at $36^{\circ} \mathrm{C}$ to guarantee the polymerization. The transversal relaxation shows two exponents after polymerization supporting the concept of partially polymerized hemoglobin. The effective transversal relaxation time decreases around $40 \%$, which can be explained by the increase of water self-diffusion coefficient 1.8 times as a main value. This result can be explained considering the effects of the agglutination process on the obstruction and hydration effects in a partially polymerized solution.
\end{abstract}

\section{Keywords}

\section{Sickle Cell Disease, Water Self-Diffusion Coefficient, $T_{2}$, NMR}

\section{Introduction}

The polymerization of hemoglobin S (HbS) is the basic molecular process in Sickle Cell Disease (SCD) [1]. An adequate understanding of the molecular processes associated to this illness is necessary in order to design different approaches for diagnostic and treatment options. Magnetic Resonance has been employed with success in the physical characterization of the HbS solution during polymerization [2]-[5] giving place to useful methods to evaluate the clinical state of the patient [6] and some potential drugs for treatment [7].

To study the water behaviour in the hemoglobin S solution is a relevant problem in SCD. Water inside hemoglobin $(\mathrm{Hb})$ solutions strongly influences the $\mathrm{HbS}$ polymerization (through concentration effect) and relates to the electrostatic interaction which stabilizes the $\mathrm{Hb}-\mathrm{Hb}$ interactions during agglutination.

How to cite this paper: Guevara, M.A.L., García-Naranjo, J.C., Mengana, Y. and Pereira, J. (2014) Hemoglobin S Polymerization Effect on Water Self-Diffusion Coefficient. Advances in Biological Chemistry, 4, 388-394.

http://dx.doi.org/10.4236/abc.2014.46044 
Particularly, the water self-diffusion coefficient $(D)$ is an important measure of the water dynamics inside the $\mathrm{Hb}$ solution and is affected by the hydration and obstruction effects according to [8]:

$$
D=D_{0}\left(\frac{(1-\varsigma \Phi)(1-f)}{1-\Phi}\right)
$$

where $D_{0}$ is the water self-diffusion coefficient of the bulk, $\Phi$ is the volume fraction occupied by hydrated $\mathrm{Hb}$, and $f$ is the fraction of water bound to $\mathrm{Hb}$. The factor $\varsigma$ depends on protein geometry [8]. The factor $(1-\varsigma \Phi)$ is related to obstruction effect and $f$ to hydration effect.

As the HbS polymerization process could affect water self-diffusion coefficient inside $\mathrm{Hb}$ solution, the main goal of this work is to compare the $D$ values before and after the agglutination process.

\section{Material and Methods}

The samples of Hemoglobin A ( $\mathrm{HbA})$ and HbS were obtained from fresh venous whole blood donated by volunteers and immediately heparinized. After removing the plasma and leukocytes, the red blood cells were washed three times with buffer saline phosphate (pH 7.4) (Sigma Chemicals Co.) and lysed by freezing [2]-[7]. The plasma and the buffer used in each washing were removed by centrifugation (2500 rpm, $10 \mathrm{~min}$ ) and decanting. After centrifuging ( $2500 \mathrm{rpm}, 10 \mathrm{~min}$ ) to remove the stroma, $500 \mu \mathrm{l}$ of hemoglobin were deposited in a nuclear magnetic resonance tube for the experiment.

The magnetic resonance measurements were performed in a magnetic resonance console (MARAN DRX, Oxford Instruments, UK). The proton transversal magnetic relaxation time $\left(T_{2}\right)$ was measured using the CarrPurcell Meiboon-Gill (CPMG) pulse sequence with a resonance frequency of $4.0353 \mathrm{MHz}$ (homogeneous permanent magnet, $B_{0}=0.095 \mathrm{~T}$ ), 10 and $20 \mu$ for $90^{\circ}$ and $180^{\circ}$ pulses respectively, 32 scans, $40 \%$ and $50 \%$ for the RF power amplifier and receiver gains respectively, $3 \mathrm{~s}$ of relaxation delay and echo time of $8 \mathrm{~ms}$.

For the measurement of $D$, the effective $T_{2}\left(T_{2 \mathrm{eff}}\right)$ was measured employing a three-magnet array unilateral magnet with extended constant gradient $\left(B_{0}=0.079 \mathrm{~T}, G=2.1 \mathrm{~T} / \mathrm{m}\right)$ [9]. The echo time was varied from 600 to $1600 \mu$ s with steps of $100 \mu$ s. Each measurement of $T_{\text {2eff }}$ was performed using CPMG pulse sequence with a resonance frequency of $3.405549 \mathrm{MHz}$. In order to maintain the same excitation bandwidth, the RF pulse length was kept at $5 \mu$ s and the RF power amplification was set at $25 \%$ and $50 \%$ for $90^{\circ}$ and $180^{\circ}$ pulses respectively. The number of scans was 512 and $3 \mathrm{~s}$ the relaxation delay. After the measurements, a plot of $1 / T_{2 \text { eff }}$ versus Tau ${ }^{2}$ was obtained, where Tau is the time between $90^{\circ}$ and $180^{\circ} \mathrm{RF}$ pulses. The slope of the straight line resulting from fitting the data was employed to calculate $\mathrm{D}$ following the expression:

$$
D=\frac{3}{G^{2} \gamma^{2}} \text { slope }
$$

where $\gamma$ is the proton gyromagnetic ratio and $\mathrm{G}$ the unilateral magnet gradient.

The samples were placed at $36^{\circ} \mathrm{C}$ to facilitate the complete polymerization process during $24 \mathrm{~h}$. Measure-

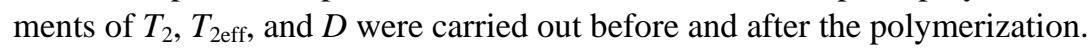

\section{Results}

The Figure 1 shows the typical curves of echo amplitude versus echo time in the HbS samples studied. It is possible to observe a stronger decreasing of the echo amplitude after polymerization, giving place to lower $T_{2}$ values than those obtained before agglutination.

Figure 2 contains the results of measuring $T_{2 \text { eff }}$ with the unilateral magnet before and after polymerization (echo time: $1200 \mu \mathrm{s}$ ). It is possible to observe a decreasing of the echo amplitude after polymerization giving place to lower $T_{2 \text { eff }}$ values than those obtained before agglutination.

Figure 3 represents the typical $D$ measurement before and after polymerization using $1 / T_{2 \text { eff }}$ behavior versus $\mathrm{Tau}^{2}$. It is possible to observe an increase of the slope after polymerization as a result of an increased $D$ value.

Table 1 shows the values of the $T_{2}, T_{2 \text { eff }}$ and $D$ after (a) and before (b) polymerization for each sickle cell patient studied. On the other hand, Table 2 summarizes the ratio between the values of these physical magnitudes before and after the agglutination process. 
$T_{2}$ measurement before and after polymerization

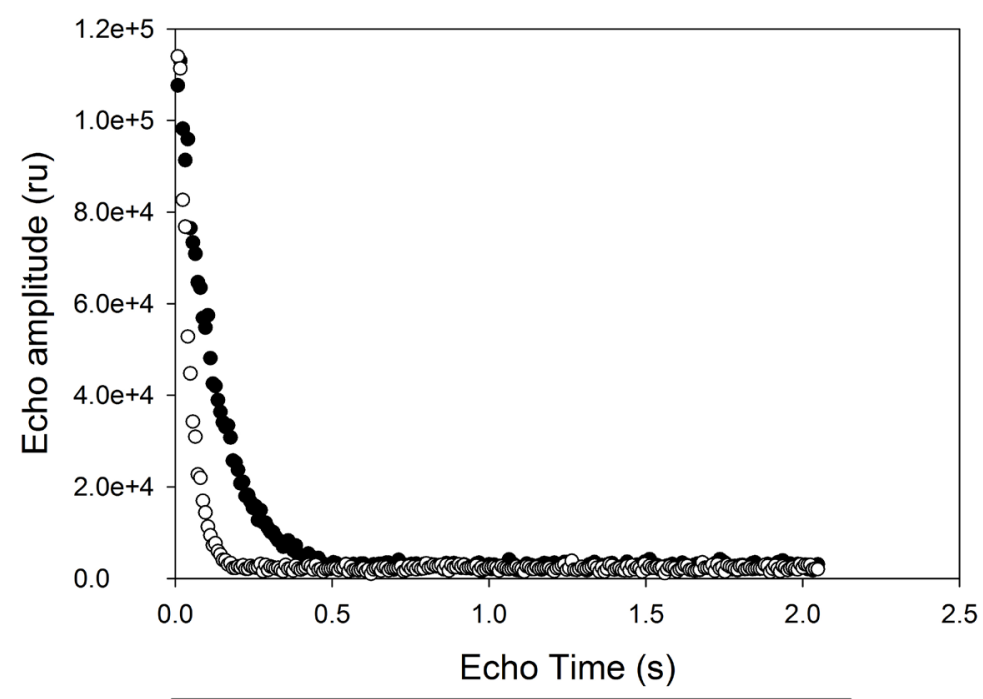

- Before polymerization $\mathrm{T}_{2}=111.7 \mathrm{~ms}$

○ After polymerization $\mathrm{T}_{2}=38.5 \mathrm{~ms}$

Figure 1. $T_{2}$ measurement before and after polymerization. The values of $T_{2}$ decrease from $111.7 \mathrm{~ms}$ to $38.5 \mathrm{~ms}$.

$\mathrm{T}_{2 \text { eff }}$ measurement before and after polymerization

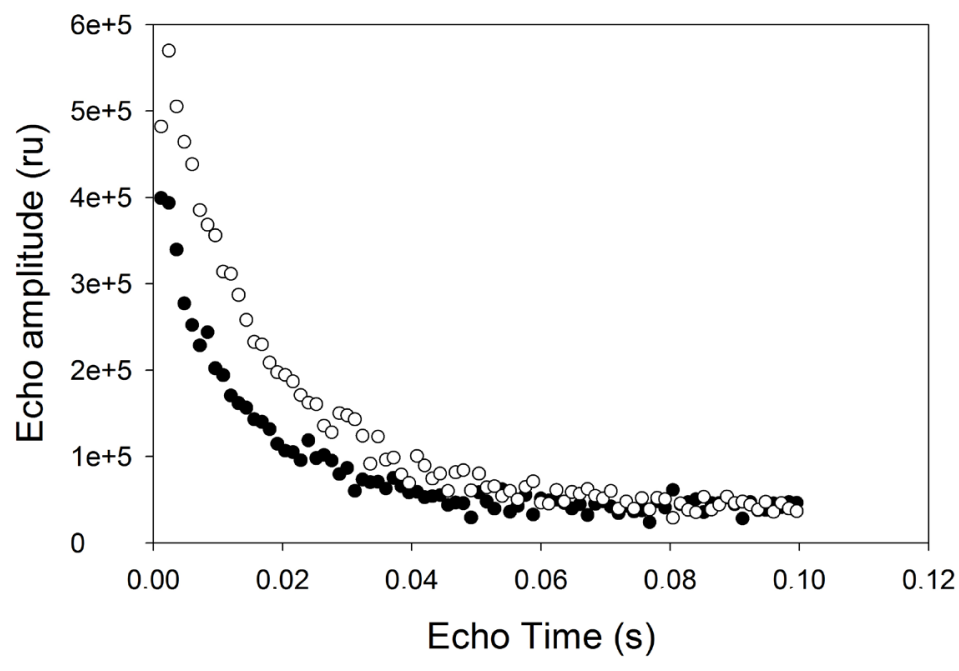

- $\quad$ After polymerization $T_{2}$ eff $=9.6 \mathrm{~ms}$

- Before Polymerization $\mathrm{T}_{2 \mathrm{eff}=14.1 \mathrm{~ms}}$

Figure 2. $T_{\text {2eff }}$ measurement before and after polymerization for tau $=600$ $\mu \mathrm{s}$. The values of $T_{2 \text { eff }}$ decrease from $14.1 \mathrm{~ms}$ to $9.6 \mathrm{~ms}$. 


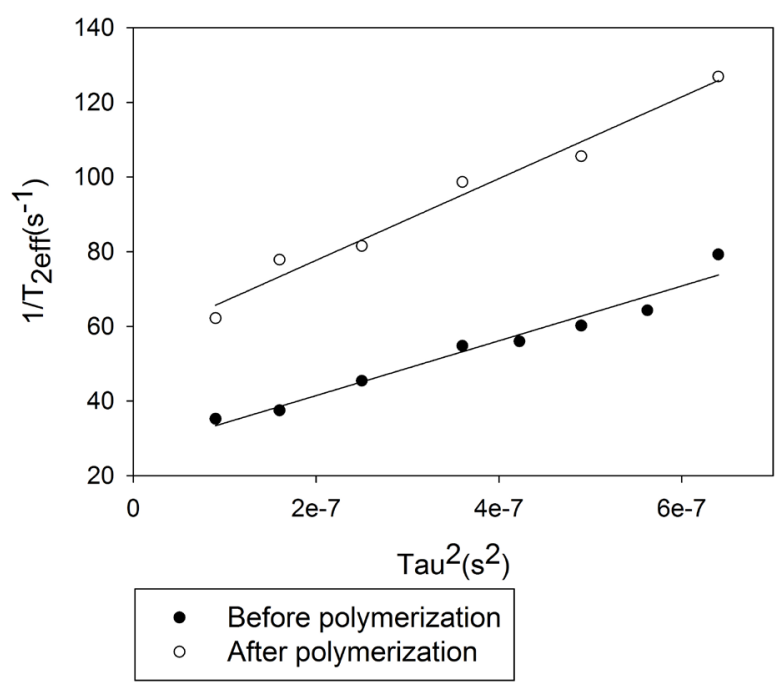

Figure 3. $D$ measurement before and after polymerization. $D$ increases from $0.62 \times 10^{-5} \mathrm{~cm}^{2} / \mathrm{s}$ to $1.04 \times 10^{-5} \mathrm{~cm}^{2} / \mathrm{s}$ with the agglutination process.

Table 1. $T_{2}, T_{2 \text { eff }}$ and $D$ after (a) and before (b) polymerization for each sickle cell patient studied.

\begin{tabular}{|c|c|c|c|c|c|c|}
\hline & Patient & 1 & 2 & 3 & 4 & 5 \\
\hline & $T_{2}^{a}(\mathrm{~ms})$ & $38.5 \pm 0.6$ & $110.5 \pm 1$ & $31.1 \pm 4.3$ & $47.9 \pm 6.5$ & $46.4 \pm 7.1$ \\
\hline & $T_{2}^{b}(\mathrm{~ms})$ & $111.7 \pm 1.4$ & $155.3 \pm 1.5$ & $113.1 \pm 1.3$ & $79.8 \pm 3.2$ & $168.4 \pm 1.4$ \\
\hline & $T_{2 \text { eff }}^{a}(\mathrm{~ms})$ & $8.5 \pm 0.2$ & $13.6 \pm 0.1$ & $8.8 \pm 0.3$ & $11.6 \pm 0.2$ & $10.1 \pm 0.2$ \\
\hline & $T_{2 \mathrm{eff}}^{b}(\mathrm{~ms})$ & $16 \pm 0.3$ & $18 \pm 0.2$ & $16.7 \pm 0.8$ & $16.1 \pm 0.2$ & $18.2 \pm 0.4$ \\
\hline & $\left(10^{-5} \mathrm{~cm}^{2} / \mathrm{s}\right)$ & $0.98 \pm 0.07$ & $0.9 \pm 0.06$ & $0.99 \pm 0.06$ & $1.17 \pm 0.05$ & $1.04 \pm 0.08$ \\
\hline$D^{b}$ & $\left(10^{-5} \mathrm{~cm}^{2} / \mathrm{s}\right)$ & $0.72 \pm 0.02$ & $0.66 \pm 0.02$ & $0.69 \pm 0.01$ & $0.34 \pm 0.03$ & $0.62 \pm 0.05$ \\
\hline
\end{tabular}

Table 2. Ratios of $T_{2}, T_{2 \text { eff }}$ and $D$ after (a) and before (b) polymerization for each sickle cell patient studied.

\begin{tabular}{cccccc}
\hline Patient & $\mathbf{1}$ & $\mathbf{2}$ & $\mathbf{3}$ & $\mathbf{4}$ & $\mathbf{5}$ \\
\hline$T_{2}^{a}$ & 0.35 & 0.71 & 0.27 & 0.27 & 0.6 \\
$T_{2}^{b}$ & 0.5 & 0.7 & 0.5 & 0.7 & 0.5 \\
$\frac{T_{\text {eff }}^{a}}{T_{\text {2eff }}^{b}}$ & 1.36 & 1.36 & 1.43 & 1.68 & 3.44 \\
$\frac{D^{a}}{D^{b}}$ & & & & & \\
\hline
\end{tabular}

\section{Discussion}

The values of $T_{2}$ observed in the Figure 1 agree with the previous report of $T_{2}$ behavior during HbS polymerization process [2] and it shows that this process is present in the samples studied. As we have demonstrated before, the observed decreasing in $T_{2}$ is the result of the increasing in the microviscosity around the $\mathrm{Hb}$ molecule, affecting its rotational movement and the rotational movement of the water strongly bound to the protein [3].

A more detailed analysis of $T_{2}$ relaxation was performed (Table 3) and significant differences were found concerning the exponential components of echo amplitude decay before and after HbS polymerization. Before polymerization one exponent was found with amplitude $A_{0}$ and relaxation time $T_{2}$. After polymerization one fast ( $A_{0}$ Fast, $T_{2}$ short) and one slow ( $A_{0}$ slow, $T_{2}$ long) exponents were found. 
Table 3. Values of the amplitude and transversal relaxation time before and after polymerization. Before polymerization one exponent was found with amplitude $A_{0}$ and relaxation time $T_{2}$. After polymerization one fast $\left(A_{0}\right.$ Fast, $T_{2}$ short $)$ and one slow ( $A_{0}$ slow, $T_{2}$ long) exponents were found.

\begin{tabular}{cccccc}
\hline \multirow{2}{*}{ Patient } & \multicolumn{2}{c}{ Before Polymerization } & \multicolumn{3}{c}{ After Polymerization } \\
\cline { 2 - 6 } & $\boldsymbol{A}_{\mathbf{0}}$ & $\boldsymbol{T}_{\mathbf{2}}(\mathbf{m s})$ & $\boldsymbol{A}_{\mathbf{0}}$ Fast $/ \boldsymbol{A}_{\mathbf{0}}$ slow $\times \mathbf{1 0 0} \boldsymbol{0}$ & $\boldsymbol{T}_{\mathbf{2}}$ short (ms) & $\boldsymbol{T}_{\mathbf{2}}$ long (ms) \\
\hline 1 & 147970.07 & 169.8 & 46.6 & 25.2 & 172.2 \\
2 & 261545 & 81.6 & 16.8 & 16.5 & 79.2 \\
3 & 72972.68 & 111.9 & 84.2 & 18.2 & 208.8 \\
4 & 151289.36 & 157.2 & 83.4 & 74.8 & 140.1 \\
5 & 121378.95 & 173.3 & 37.6 & 25.5 & 164.7 \\
\hline
\end{tabular}

The results in Table 3 show the splitting of the water inside the HbS solution in two populations after polymerization. One population with faster transversal relaxation ( $T_{2}$ short), which should include the water bound to the polymerized hemoglobin, and another population with slower transversal relaxation ( $T_{2}$ long) including the water bound to free $\mathrm{HbS}$ in the solution. This result characterizes the hemoglobin solution studied as: Partially Polymerized, which agrees with our previous physical characterization of the HbS polymerization [3].

Using the model of polymerization developed by Cabal and Ruiz [10], and updated by Cabal and Lores [11], under absence of crystalization and when the stability condition is reached, the HbS polymerization can be described as follows:

$$
y=\frac{P[x(t)]}{b}
$$

where $y$ represents the polymer concentration, $x(t)$ the deoxygenated HbS concentration, $P$ the polymerization function and $b$ the de-polymerization coefficient.

As our experiment was performed under spontaneous deoxygenation conditions, reaching only around $50 \%$ of deoxygenated hemoglobin during the time of the experiment [11], just a limited amount of polymers will be obtained together with another amount of $\mathrm{HbS}$ molecules remaining free inside the solution.

As we have used a strong magnetic field gradient in our experiment $(G=2.1 \mathrm{~T} / \mathrm{m})$ to determine $T_{\text {2eff, }}$, the values observed in the Figure 2 are mainly related to the difusional movement of this water between its bond site and the bulk [12]:

$$
\frac{1}{T_{\text {2eff }}}=\frac{D G^{2} \gamma^{2}}{3} \operatorname{tau}^{2}
$$

Considering this point of view; to study the polymerization process in one inhomogeneous magnetic field with strong and constant magnetic field gradient, as is the case of the results reported in this work, is another tool to study the agglutination process, focusing our interest in the translational movement of the water bound to hemoglobin.

It is possible to conclude from Figure 2 (and Table 1 and Table 2) that the polymerization of HbS causes a significant decreasing of $T_{2 \text { eff }}$; which should be related with the increasing of $D$ according to Equation (4). The mentioned behavior of $D$ was confirmed during our experiment and it is summarized in Figure 3 and Table 1 and Table 2. It is possible to observe how the self-diffusion coefficient of water increases in all the sickle cell patients studied 1.8 times as a main value. The same experiment performed in $\mathrm{HbA}$ shows a constant behavior of $D$ before $\left(D_{b}=(0.98 \pm 0.1) \times 10^{-5} \mathrm{~cm}^{2} / \mathrm{s}\right)$ and after $\left(D_{a}=(0.98 \pm 0.2) \times 10^{-5} \mathrm{~cm}^{2} / \mathrm{s}\right)$ the agglutination process, which confirms the polymerization as the origin of the change of this parameter observed in HbS.

The values of $D$ obtained for $\mathrm{Hb}$ solution match with the previous reports of translational water self-diffusion coefficients in hemoglobin solutions [13]. Considering our HbS solution became a partially polymerized solution, the increase of $D$ could be explained, according to Equation (1), considering the following process:

- The $\mathrm{Hb}-\mathrm{Hb}$ interactions during $\mathrm{HbS}$ polymerization have the same origin that those supporting the water binding to $\mathrm{Hb}$ (electrostatic interactions like: Hydrogen bridge interaction and Van der Waals interaction) [1] [11]. Then, to guarantee the electrostatic $\mathrm{Hb}-\mathrm{Hb}$ interaction which stabilizes the polymers, the HbS polymerization could decrease the number of sites available for water binding to $\mathrm{Hb}$, causing a decreasing of $f$ and the increasing of $D$. 
- The HbS polymerization provokes the appearance of long fibers inside the hemoglobin solution, which could increase the obstruction effect in the region of the partially polymerized sample where water is located near the fibers (Polymerized region). Nevertheless, as the polymer's growth results from the initial and constant number of $\mathrm{HbS}$ molecules, in the other region where the water is located far from the fibers (Free region) the obstruction effect could decrease. Starting from the data in Table 3, in our partially polymerized solution the free region is dominant. It causes the decreasing, as average, of the obstruction effect provoking the increasing of $D$.

\section{Conclusion}

The concept of partially polymerized hemoglobin, under spontaneous deoxygenation conditions, is supported through the presence of two exponents characterizing the magnetic transversal relaxation of HbS samples. A new approach to study HbS polymerization process has been presented, starting from the study of $T_{2 \text { eff }}$ and $D$ in one inhomogeneous and portable magnetic field with one strong and constant static magnetic field gradient. The $T_{\text {2eff }}$ values decrease around $40 \%$, which can be explained by the increasing of $D 1.8$ times as a main value. The increasing of $D$ can be explained through the decreasing of the hydration and obstruction effects as a result of the agglutination process in a partially polymerized hemoglobin solution.

\section{Acknowledgements}

This work has been supported by the Belgian Development Cooperation through VLIR-UOS (Flemish Interuniversity Council-University Cooperation for Development) in the context of the Institutional University Cooperation program with Universidad de Oriente.

The authors also want to thank the MRI Research Centre of the University of New Brunswick, Fredericton, Canada, for all the support received to finalize this work.

\section{References}

[1] Ferrone, F.A., Hofrichter, J. and Eaton, W.A. (1985) Kinetics of Sickle Hemoglobin Polymerization. II. A Double Nucleation Mechanism. Journal of Molecular Biology, 183, 611-631. http://dx.doi.org/10.1016/0022-2836(85)90175-5

[2] Lores, M. and Cabal, C. (2005) Proton Magnetic Relaxation Process during the Polymerisation of Hemoglobin S. Applied Magnetic Resonance, 28, 79-84. http://dx.doi.org/10.1007/BF03166995

[3] Lores, M., Cabal, C., Nascimento, O. and Gennaro, A.M. (2006) EPR Study of the Hemoglobin Rotational Correlation Time and Micro Viscosity during the Polymerization of Hemoglobin S. Applied Magnetic Resonance, 30, 121-128. http://dx.doi.org/10.1007/BF03166986

[4] Cabrales, Y., Lores, M. and Machado, Y. (2008) Deuterium Magnetic Relaxation Process during the Polymerisation of Hemoglobin S. Applied Magnetic Resonance, 33, 207-212. http://dx.doi.org/10.1007/s00723-008-0074-z

[5] Falcón, J.E., Rodi, P., Lores, M. and Gennaro, A.M. (2010) Spin Labels Studies of the Hemoglobin-Membrane Interaction during Sickle Hemoglobin Polymerization. Applied Magnetic Resonance, 38, 443-453. http://dx.doi.org/10.1007/s00723-010-0138-8

[6] Fernández, A., Cabal, C., Lores, M., Losada, J. and Pérez, E. (2009) Sickle Cell Disease "Painful Crisis” and "Steady State” Differentiation by Proton Magnetic Resonance. Hemoglobin, 33, 206-213. http://dx.doi.org/10.1080/03630260903039578

[7] Fernández, A.F., Cabal, C., Losada, J., Álvarez, E., Soler, C. and Otero, J. (2005) In Vivo Action of Vanillin on Delay Time Determined by Magnetic Relaxation. Hemoglobin, 29, 181-187. http://dx.doi.org/10.1081/HEM-200066305

[8] Baranowska, H.M. and Olszewski, K.J. (1996) The Hydration of Proteins in Solutions by Self-Diffusion Coefficients NMR Studies. Biochimica et Biophysica Acta, 1289, 312-314. http://dx.doi.org/10.1016/0304-4165(95)00141-7

[9] García, J.C., Mastikhin, I.V., Colpitts, B.G. and Balcom, B.J. (2010) A Unilateral Magnet with an Extended Constant Magnetic Field Gradient. Journal of Magnetic Resonance, 207, 337-344. http://dx.doi.org/10.1016/j.jmr.2010.09.018

[10] Cabal, C., Libório, A.M. and Ruiz, A.I. (2007) A Model of the Molecular Aggregate Processes of Hemoglobin S. Absence of Crystallization. Mecánica Computacional, 26, 3361-3369.

[11] Lores, M. (2005) Magnetic Resonance Study of the Magnetic Interaction Process and Molecular Mobility during HbS Polymerization. Ph.D. Dissertation, University of Oriente, Santiago de Cuba.

[12] Kimmich, R. (1997) NMR Tomography, Diffusometry, Relaxometry. Springer, Berlin. 
[13] Heinz-Jürgen, S., Berthold, K., Gerd, H., Owerdieck, C. and Redhardt, A. (1993) Rotational and Translational Water Diffusion in the Hemoglobin Hydration Shell: Dielectric and Proton Nuclear Relaxation Measurements. Biophysical Journal, 65, 1486-1495. http://dx.doi.org/10.1016/S0006-3495(93)81217-7 
Scientific Research Publishing (SCIRP) is one of the largest Open Access journal publishers. It is currently publishing more than 200 open access, online, peer-reviewed journals covering a wide range of academic disciplines. SCIRP serves the worldwide academic communities and contributes to the progress and application of science with its publication.

Other selected journals from SCIRP are listed as below. Submit your manuscript to us via either submit@scirp.org or Online Submission Portal.
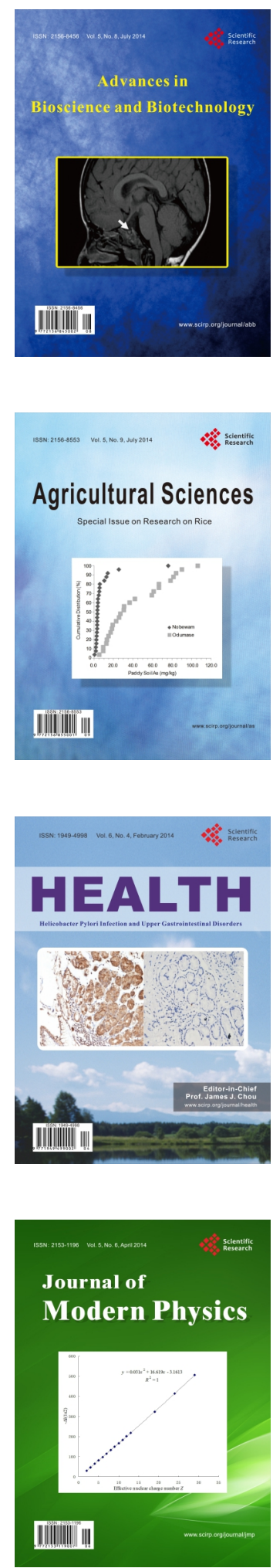
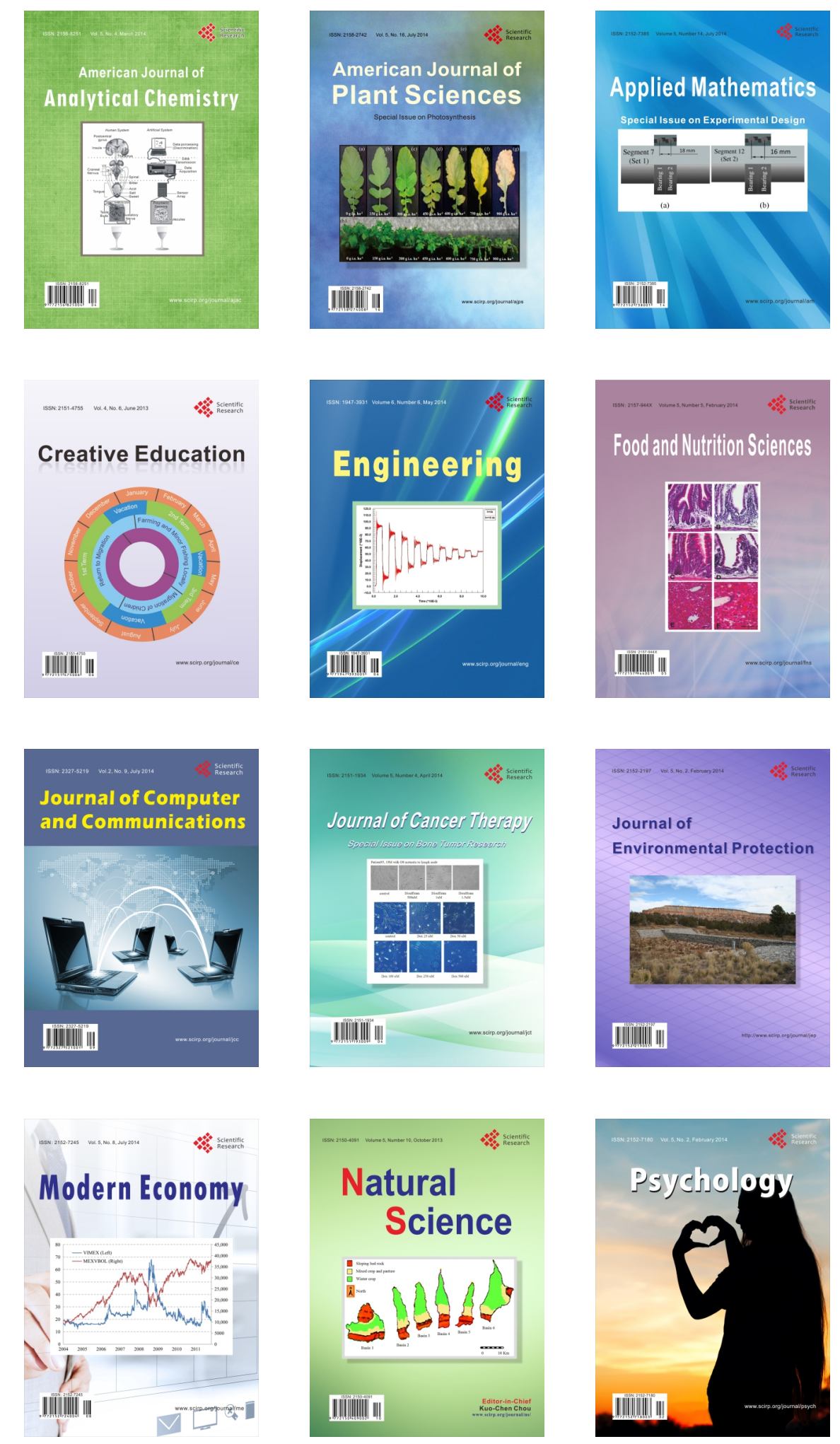